\title{
A Supplementary
}

\section{A.1 Data Source}

The MAREA Gait Database is available at: http://islab.hh.se/mediawiki/ Gait_database. The Human Gait Database(HuGaDB) is available at: https : //github.com/romanchereshnev/HuGaDB.

\section{A.2 Coverage proportion curves of Principle System-States}

\section{(A)}

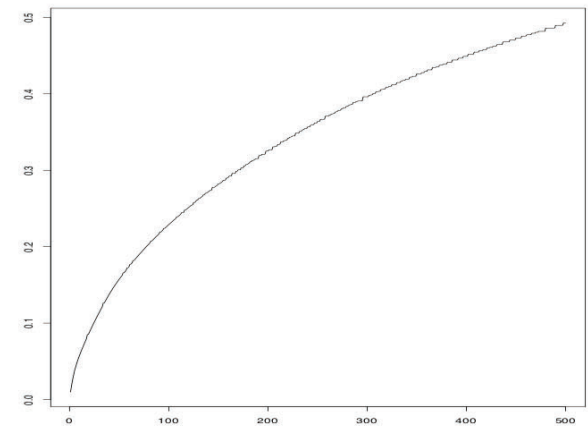

(B)

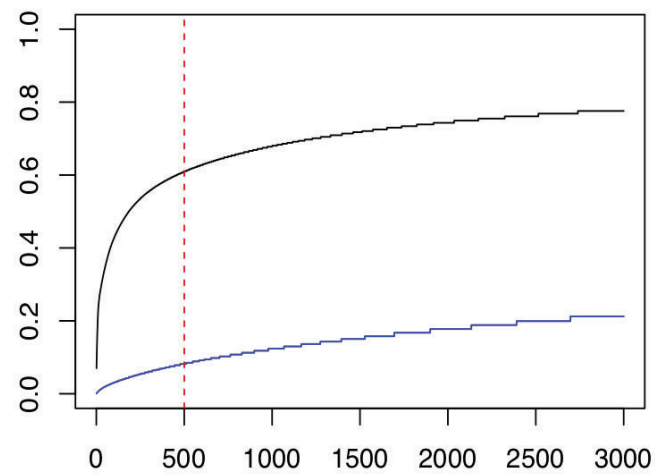

Figure 9: (A): $r\left(N^{*}\right)$ v.s $N^{*}$ from 9-dim gait time series from 3 sensors fixed at Left foot and Right foot and wrist among 10 subjects in MAREA database. The triple coding is based on $\alpha=0.3$ and $\beta=0.7$ quantiles.

(B): $r\left(N^{*}\right) v . s N^{*}$ based on 18-dim gait time series derived from 6 sensors fixed to left and right thighs, shines and feet in HuGaDB database. The black curve is pertaining to the triple coding based on $\alpha=0.1$ and $\beta=0.9$ quantiles, while the blue curve is based on $\alpha=0.3$ and $\beta=0.7$ quantiles 

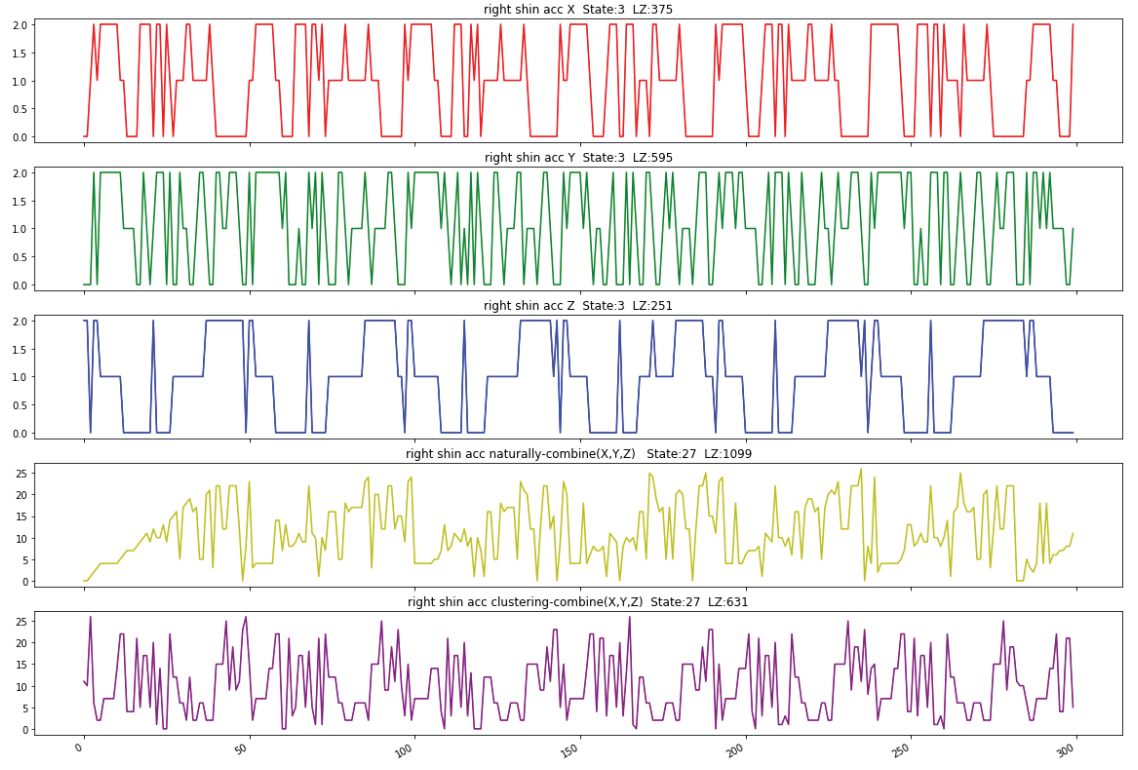

Figure 10: From top to bottom, code each accelerometer time series from right shine separately and combine them into one sequence in two different ways; one is a natural way of combination (the second last to the bottom), the other is our clustering-based combination (the last); ours has less LZ complexity around a half compared with the natural way; the seasonal pattern is shown clearer and more rhythmic in our method 

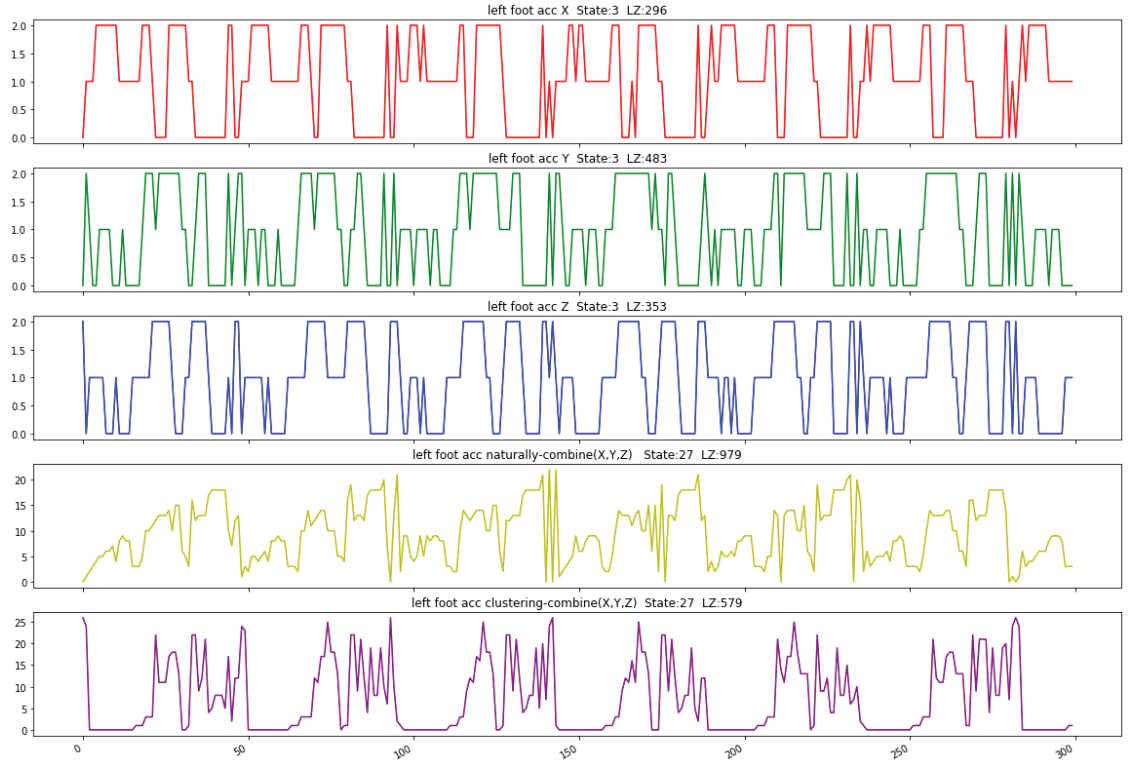

Figure 11: From top to bottom, code each accelerometer time series from left foot separately and combine them into one sequence in two different ways; one is a natural way of combination (the second last to the bottom), the other is our clustering-based combination (the last); ours has less LZ complexity around a half compared with the natural way; the seasonal pattern is shown clearer and more rhythmic in our method 